\title{
Community-based psychodynamic group psychotherapy for treatment-resistant schizophrenia
}

\author{
Giuliano Aiello \& Sheraz Ahmad
}

\begin{abstract}
SUMMARY
We describe the work of a psychodynamic psychotherapy group for individuals affected by chronic schizophrenia in our community-based rehabilitation and recovery service. Despite presenting with negative symptoms, over time patients made good use of the psychodynamic setting: they attended, expressed emotions, made connections and reflected within the group on their personal experience of mental ill health. We discuss the rationale and indications for psychodynamic psychotherapy for individuals with severe and enduring mental illnesses. We also reflect on the challenges we faced working psychodynamically with psychosis and on the necessary adaptation of the approach to work with chronic psychosis. We present vignettes from group sessions to illustrate themes that we identified in the therapy, making links with the literature. We conclude that the unstructured setting of the psychodynamic group approach can help individuals affected by most severe forms of psychosis to make sense of their experiences and use the space.
\end{abstract}

\section{LEARNING OBJECTIVES}

- Understand the rationale for psychodynamic group interventions for psychosis.

- Understand what adaptations of psychodynamic approaches are necessary to work with patients with psychosis.

- Change attitudes toward offering psychodynamic interventions for psychosis.

\section{DECLARATION OF INTEREST}

None.

Psychoanalytic views on psychosis have changed over time. Freud at first argued that, in psychosis, the libido (emotional investment) had been withdrawn from the outside world and consequently no transference was possible (Freud 1914). He subsequently moderated his view and, later on, psychoanalysts such as Rosenfeld (1965) and Bion (1957) highlighted the role of transference in psychosis and explored the nature of psychotic transference and ways of working with it psychodynamically. Bion (1957) found that the psychotic relationship with the analyst was characterised by premature and intense feelings and heavy use of projective identification. He also made the distinction between psychotic and neurotic parts of the personality in individuals affected by psychosis. Lucas (2008) explored the implications of this idea in the clinical and psychotherapy context. He described how, listening to the patient in a therapeutic clinical setting, the clinician can identify neurotic and psychotic components in the patient's speech and facilitate a dialogue between the two. Recent interest in a psychoanalytic approach to psychosis suggests that it has a role in general psychiatry, helping clinicians to make sense of the chaotic presentation of chronic psychosis and offering the patient a space to reflect freely on their experience (Martindale 2007; Lucas 2008; Rosenbaum 2013).

\section{Group psychotherapy for psychosis}

\section{History}

Group psychotherapy for psychosis has a long tradition dating back to the 1930s. The first psychoanalytic formulation of group psychotherapy dates back to the 1940s with Foulkes (1957). Over time, psychodynamic group psychotherapy has played a role in the treatment of psychosis (Chazan 1993), although little evidence has been produced to support its effectiveness. Overall evidence suggests that group psychotherapy could be a useful tool alongside medication (de Bosset 1991) and that focusing on present emotions and interactions is more beneficial than uncovering unconscious feelings (Kanas 1986, 1996).

Individuals suffering from chronic psychosis in the community often face significant social isolation (Reininghaus 2008). Group psychotherapy can offer these people a space to share personal experiences and an opportunity to mutually observe and reflect on emotions, thoughts and feelings (Kanas 1996; de Chavez 2000). Instillation of hope, universality, cohesiveness and

\section{ARTICLE}

Giuliano Aiello is a specialty doctor on the City \& Hackney Speciality Doctors Rotation, East London NHS Foundation Trust. Sheraz Ahmad is a consultant psychiatrist with City \& Hackney Community Rehabilitation and Recovery Service, East London NHS Foundation Trust. Both authors have a special interest in psychosis, psychosocial intervention and psychodynamic psychotherapy. Correspondence Dr Giuliano Aiello, City \& Hackney Specialist Addiction Unit, East Wing, Homerton Hospital, Homerton Row, London E9 6SR, UK. Email: giuliano.aiello@ eastlondon.nhs.uk 
expressiveness are the most consistent benefits reported in the literature (Maxmen 1973; Kanas 1986; Yalom 1983; de Chavez 2000). It has been suggested that, in a group, psychotic transference can be less intense because the patient has the opportunity to see the therapist as a real person and thus emotion can be dealt with and explored more safely (Gabrovsek 2009).

\section{Current use}

There is a strong tradition of psychoanalytic intervention for psychosis in Scandinavian countries and it remains an important approach among the treatments offered. Several studies describe the valuable role of this intervention within integrated treatment for psychosis (Rosenbaum 2005, 2013; Seikkula 2006). Nevertheless, in the past two decades, partly owing to lack of evidence of efficacy, psychoanalytic approaches to psychosis treatment in the UK have fallen out of favour with general psychiatry.

Currently, the only psychological therapy recommended by the National Institute for Health and Care Excellence (NICE; 2014) for use in schizophrenia is cognitive-behavioural therapy (CBT). A set of CBT interventions has been developed for psychosis, involving a combination of psychoeducation and social and functional adaptation skills training (Tarrier 2002; Jones 2012). This type of structured and symptomfocused intervention has been successful in working with patients with psychotic illnesses (Tarrier 2002), although recent meta-analyses and literature reviews raise questions about its effectiveness (Turkington 2003; Jones 2012; Jauhar 2014).

In this context of uncertainty regarding the effect of specific psychotherapy interventions, individuals with chronic psychosis (particularly treatmentresistant schizophrenia) may receive only repeated cycles of CBT and may be excluded from the possibility of trying different approaches. A recent review of psychological therapies for psychosis explored the literature on several cognitive and supportive psychotherapies suggested by experts in the field, but a psychodynamic approach was not mentioned at all (Bridle 2013).

\section{Psychodynamic psychotherapy in action}

In this article, we report our experience of setting up and running a psychodynamically informed psychotherapy group for patients on a community rehabilitation service case-load (all affected by chronic, treatment-resistant forms of schizophrenia). Our intention was to offer a psychotherapy to our patients without any exclusion criteria. Inspired by Lucas (2008), we set up a space in which patients could talk and share safely, with few demands made of them. We expected to be exposed to a lot of bizarre and psychotic material. We hoped to address that material in a psychodynamic way and we also hoped that listening to psychosis would allow emotions to be expressed and explored.

\section{Group setting and structure}

The group meets once a week at the community rehabilitation service base. Meetings last 1 hour and keep to the same time and day each week. The group first met in July 2011, with the intention of continuing throughout the year with no breaks. So far we have had three breaks: two for team awaydays and one for a consultant interview. Currently co-facilitated by the consultant psychiatrist and a middle-grade psychiatrist from the team, the group has also had periods of input from a second consultant psychiatrist, a junior doctor and a social worker. It is open to all patients under the care of the team. It is an open-attendance group (i.e. patients are free to join and leave the group without any minimum time commitment) with a maximum of 10 attendees per session (this was estimated with room size in mind). If fewer than three patients attend, we agreed to shorten the meeting to $30 \mathrm{~min}$.

Material for discussion is brought by the patients, but few demands are made of attendees. A high level of demand is made of the facilitators. For example, classic free-floating discussion as described by Foulkes (1957) is encouraged, but facilitators are expected to be more proactive than in classic psychodynamic groups by offering connections, ideas and containment to the group.

Attendance and notes of each session are recorded at the end of the session. If a patient attending the group is going through a difficult time, the content of the session may be shared and reflected on with the rest of the team, to help them gain a better understanding of the patient and their crisis.

Both facilitators have a special interest in psychodynamic psychotherapy: the consultant psychiatrist has broad experience in facilitating psychodynamic group psychotherapy, and the middle-grade doctor has conducted individual cases of psychodynamic psychotherapy. The group has also been co-facilitated by clinicians with no background in psychodynamic psychotherapy, but it is important that at least one of the facilitators is familiar with the psychodynamic framework.

Supervision is a necessary component of psychological intervention and this group is no different 
from other modalities: supervision helps contain and reflect on facilitators' emotional reactions to the group and clarifying the group dynamics. Facilitators attend monthly supervision with the consultant psychiatrist in psychotherapy for the local service. These meetings also provide a structured space for facilitators to develop the group's narrative.

We had no specific inclusion or exclusion criteria for group members, and care coordinators were encouraged to offer participation to all the patients on our case-load. All our patients have a long history of schizophrenia or schizoaffective disorder. Box 1 shows group attendance over a 2-year period. Care coordinators' involvement was crucial in encouraging initial attendance. In the end, it was difficult to predict who could/would attend and benefit from the group - however, we acknowledge that our patients share particular characteristics and so a group like this in a more general setting would differ greatly.

\section{Vignettes from our group sessions}

We present some session vignettes from different times in the group's history and the themes emerging. We thank the patients involved for permitting us to discuss their experiences here.

$\mathrm{R}$ is a middle-aged African-Caribbean man affected by paranoid schizophrenia. He was severely physically disabled 30 years ago during a psychotic crisis and does not remember what happened. Although he attends regularly and is protective towards the group, his thinking is very concrete and he seldom accepts reflections.

$\mathrm{L}$ is a middle-aged White British man affected by paranoid schizophrenia who attends the group regularly. For several months after joining he did not participate in discussions, but more recently he has started to share with the group his mistrust and his fear of talking to psychiatrists.

$\mathrm{J}$ is a middle-aged African-Caribbean man affected by schizoaffective disorder, who recently moved to independent accommodation. He tends

BOX 1 Attendance at the group

\begin{tabular}{ll}
\hline $\begin{array}{l}\text { July } 2011 \text { to June } 2013 \\
\text { Total number of attenders }\end{array}$ & 108 sessions \\
(at least one session) & 25 \\
Average number of attenders & \\
per session & 4 \\
Regular attenders & 8 \\
No one attended & 1 session (second \\
session) &
\end{tabular}

to have poor contact with other people, and is very open about his grandiose and bizarre delusional beliefs. He attended the group only for four sessions.

\section{Session A (first half): $R, L$ and $J$ attended; $G A$ and $S A$ were the facilitators}

The first half of the group was dominated by J, who talked about various psychotic phenomena. SA reflected to $\mathrm{J}$ that he had told us previously that he would not be returning to the group. J reminded us that he had said there was a 'small possibility' he would be back and he was curious about whether the 'conundrum' he left us with last time had been resolved. SA asked him to remind the group: he told us about the Arthurian circle with a 'dangerous seat' which could only be sat in by a 'truly great Knight'. He pointed out that we too were sat around in a circle and he wondered which was the 'dangerous seat'. He then talked about having not achieved anything in his life and feeling like a failure and the idea of being a 'mad Don' and whether one could be the 'maddest' and whether that was an achievement in itself. GA noted that when the connection was made between angry feelings towards his father and the flight into psychosis J quickly changed subject and pursued paranoid thinking. The observation was gently repeated and $\mathrm{J}$ was able to reflect a little on painful memories without actually telling the group what took place.

\section{Talking about psychosis rather than being psychotic}

For J, the group intervention was to explore the healthy part of his mind by pursuing a neurotic reflection on the psychotic material he brought. The idea of distinguishing between a psychotic and a non-psychotic (neurotic) part of the personality (Bion 1957; Lucas 2008) had a significant influence on our therapeutic work and helped us to make sense of our role in the unstructured setting when psychotic material emerged in the sessions. Reflecting with the group on how difficult it was to understand his psychotic material, J acknowledged the possibility that his psychotic experiences could, in part, be a way of avoiding more painful memories, in particular regarding his relationship with his father.

Psychotic material often came to the surface spontaneously in the group. Lucas suggests that denial is one of the main features of psychosis; for example, people with psychosis try to deny the psychotic nature of their experiences when confronted with it. At the beginning, our group reacted to psychotic material or bizarre behaviour with complete denial of the psychosis, refusing to make any comment on it and trying to normalise it or change the subject. Over time, psychotic communication became more tolerable and contained in the group and the shift from being psychotic to talking about psychosis became more 
possible. The literature recognises the dangers of over-interpreting psychotic material, especially in believing that complete sense can be made of nonsense (Fromm-Reichmann 1948).

We found that dealing with psychosis as a part of human experience rather than as an illness helped the group to contain the anxiety and overcome the instinctive tendency to deny psychosis well described by Lucas. When the group started to feel more comfortable discussing psychosis, making minimal, gentle interpretations and exploring different perspectives became possible.

\section{Session A (second half)}

$\mathrm{J}$ said that sometimes we have painful memories we do not want to remember. SA presented this to the group and wondered with $\mathrm{R}$ about his chronic difficulties with remembering. Although no one made specific reference to his physical impairment, $\mathrm{R}$ was keen to think about his wish not to know what happened. Indeed, he described how people on the street would sometimes stop him and tell him that they knew what happened to him, but he did not believe them. $\mathrm{R}$ asked us what happened to him and we wondered with him whether he would be more likely to believe the story if a doctor told him. Both GA and SA felt a strong wave of emotion when $\mathrm{R}$ talked about the possible versions of events and whether he could have been involved in a fire and also a recent idea he had that his mother might have been present.

During the group, L remained cautious about contributing but was clearly engaged. He was keen to consider whether thinking about certain things could be damaging; SA reflected back to him whether he worried about certain thoughts hurting him. At the end, when SA linked the two halves of the session, J was able to reflect that he too had some blank memories of things that had happened in the past and he also felt he knew how dangerous fire could be.

The group also reflected at the end about the great discharge of emotion during R's story and about what this kind of disclosure might feel like for him despite him telling us that he felt 'fine'. There was a strong theme about not wanting to remember and the risk of remembering something and how it would feel. Although $\mathrm{R}$ did not express a wish to know what had really happened, SA did wonder with the group what the motivation for his regular attendance might be.

\section{A concrete space}

$\mathrm{R}$ was our most regular attender at the group. In the course of the therapy we repeatedly offered him opportunities to discuss the meaning of his attendance and attachment to the group, and for a long time his comment was that coming to the group was just 'something to do and a reason to go out'. As we see in the previous and following session vignettes, after nearly 2 years, $\mathrm{R}$ spontaneously talked about his inability to remember how and why he was injured and the group empathised with him and reflected on it. This change was emotionally intense for R. Progressively, his concrete thinking partially gave way to windows of spontaneous feeling and active engagement; the group perceived the difficulty $\mathrm{R}$ was going through in recalling such painful memories and offered him containment and empathic understanding.

Working with chronic schizophrenia means dealing with negative symptoms. Despite our repeated attempts, for a long time concreteness presented an obstacle to reflecting on attachment with the group. Interestingly, this was particularly true for $\mathrm{R}$ and $\mathrm{L}$, who attended the group from very early on and for a long time. They also arrived for the group at least an hour in advance, demonstrating their attachment but finding it difficult to reflect on what it might mean.

Concrete thinking is an obstacle to engagement with psychodynamic psychotherapy because it allows no space for reflection. Smith (1999) points out that, in the group, responses such as emotionless listing and repeating can represent an 'attack [on] the therapist's ability to think, and [that] boredom, which represents a return to deadness, is a frequent experience'. A common challenge for us as group facilitators was diverting or interrupting lists of things such as places people had lived, lists of different food and what people bought in shops. Nevertheless, we held some optimism about the possibility of processing emotional material within a concrete space in an unstructured way. In our group we offered reflections and interpretations without expectation that the patient would readily take them on board. We often had the impression that we were thinking for the group rather than with the group, then being surprised when someone, usually rather abruptly, expressed feelings more eloquently than expected. In the end, we found that this was useful and containing and that even the concrete attachment had meaning and could be worked through.

\section{Session B: $R$ \& $L$ attended; GA and SA were the facilitators}

The session began talking about how both $\mathrm{L}$ and $\mathrm{R}$ had been coming to the group for a very long time but how in some ways they maintained a distance from the group, $\mathrm{L}$ by refusing to share what's in his mind for fear of repercussions, and $\mathrm{R}$ by claiming that he forgets everything. SA reflected on the observation that, despite regular attendance, $\mathrm{L}$ has been consistently reluctant to share what is in his mind. L expressed an anxiety about talking about things that other people would think are 'unbelievable'. He was clear that it was difficult and distressing to not be believed and then for it to be interpreted as a sign of mental illness. 
GA observed that, over time, L had been able to open up more and tell us about his opinions and share some everyday difficulties. SA then wondered whether hearing other people in the group talk about their unbelievable experiences had influenced L; L went on to talk about having seen a group member who was not present today at the cinema watching Life of Pi. L was determined to tell us about the film, thus avoiding the subject that we were discussing He told us about the film in a very straightforward fashion, focusing on the actual events on the screen, the difficult relationship between the boy on the boat and the tiger. This seemed to spark some talk about tigers in films with $\mathrm{R}$, and although GA and SA were trying to interpret L's material, $\mathrm{R}$ was determined to tell us about other films that had tigers in them, such as The Jungle Book. SA then reflected back to $\mathrm{R}$ that his memory was not as bad as he would have us believe and we acknowledged together how there may be a wish to forget or remember certain things. GA then talked about how the difficult relationship between the boy $(\mathrm{Pi})$ and the tiger and the wariness that the boy has of the tiger may tell us something about L's contact with the group. SA raised the ending of the film, when the viewer realises that the animals on screen were in fact representing people from memory and the story had been remembered in a way that was more tolerable by the protagonist. SA noticed how this tied together some of the central difficulties that $\mathrm{R}$ and $\mathrm{L}$ had in the group. $\mathrm{L}$ then wondered whether he had not understood the film correctly and felt that SA had probably got it right. It was unclear whether he took on board SA's comment.

$\mathrm{R}$ started to share his own unbelievable experiences unprompted for the first time. He talked about voices and telepathy and how he had been forced to change his view of these experiences. He wondered whether the voices he heard might be symptoms of illness rather than instances of telepathy. When we asked L how he felt about $\mathrm{R}$ discussing this openly, L started laughing warmly and we all laughed together, and $\mathrm{L}$ said that he didn't know what to say about R's experiences. L was clear that telepathy is real and that there is something quite unfair about unusual experiences being interpreted as illness. He felt that what doctors sometimes called voices were in fact forms of mental communication. The tone in the room throughout the session was warm and intimate and it felt as though the group was able to make some headway, especially with the powerful imagery in symbolism that was introduced by the film. Both $\mathrm{L}$ and $\mathrm{R}$ were animated and involved.

\section{Transference with psychiatry and psychiatrists}

$\mathrm{L}$ was a regular attender at the group, but for a long time his participation was minimal and he used to remain largely silent throughout the sessions. In our vignette from Session B we see how, after several months of silence, he began to talk, sharing his fear of being misunderstood and considered 'mad' by others. We invited him to reflect on his difficulties in talking to us as well as on his fear that his beliefs could be misunderstood by psychiatrists. Together with the group, we reflected on the meaning of other patients' psychotic material and on how judgement or misjudgement arise. L progressively started to join discussions regarding magical thinking and telepathy and to guess about therapists'/ psychiatrists' opinions on these. Observing over the months how the group and the therapists treated psychotic material nonjudgementally, L found a way to express his fear of being judged by us and his wish to leave psychiatry.

In our position as group facilitators, we are both clinicians responsible for medical care and therapists, and this has an important effect on the transference feelings that the therapist can elicit in the patient. Psychiatrists usually play an important role in the lives of patients with a long history of mental illness (Hughes 2000), a role perceived at different times as caring or intrusive. We can often observe how these feelings are reactivated during a psychotic relapse and projected onto the psychiatrist with overwhelming intensity. Psychodynamic psychotherapy groups offer a space in which patients' personal feelings towards psychiatry can be safely shared, contained and eventually become more tolerable. There may also be the opportunity to express anger, disappointment and fear in relation to their psychiatrists.

\section{Implications for practice}

\section{Psychodynamic groups do help in psychosis}

The work of the group demonstrates how severely ill patients with psychosis, usually considered unsuitable for psychotherapeutic interventions, managed to use the space in a personal and meaningful way. Reactions to the group setting and material brought were different for each participant, reflecting individual experiences and dramas related to a life with psychosis. Nevertheless, the group managed take up a position next to the patient most of the time, even when confronted with particularly puzzling and emotive material, as in the case of R.

\section{Engagement: a problem that can be overcome}

Usually, patients need time to get used to the unstructured setting of a psychodynamic group and actively contribute to it. In a first stage, they may relate solely to the therapist and resist working as a group (Foulkes 1957). This stage was particularly prolonged with our patients, and the stability and cohesion that the group sometimes achieved was always fragile. Whenever an event disrupted the balance of the group, such as a new member joining, the group tended to regress to a stage of lesser cohesion. In general, when more people attended, it was difficult to recreate the 
MCO answers

$\begin{array}{lllll}1 \mathrm{a} & 2 \mathrm{~d} & 3 \mathrm{~d} & 4 \mathrm{a} & 5 \mathrm{~b}\end{array}$ warmth and empathy described in the vignettes presented here. There was a difference in tone when more people attended, but like all group work we took the position that, however many came, we would make use of the sessions.

\section{The difficulty of eliciting emotional response in psychosis}

One perspective on group psychotherapy suggests that it works by stimulating empathy (Foulkes 1957; de Chavez 2000). The ability to show interest and empathy for others is not a spontaneous process in patients affected by schizophrenia (Haker 2009). Impairment of empathy and social understanding and an inability to mirror the emotions of others are often severe in chronic schizophrenia (Penn 2008; Haker 2009). Indeed, they are possibly very close to the organic substrate of the illness (McCormick 2012). This is probably what brought Freud to think that patients with psychosis are not suitable for psychotherapy because of their lack of transference and narcissistic withdrawal. As facilitators, we had to constantly observe, stimulate and elicit emotion in the group and we were often left feeling bored and disconnected. Occasionally, an emotional response would surface, often intensely. For example, it took $\mathrm{R}$ and $\mathrm{L}$ more than a year to start to share personal worries, and this sharing eased tension and allowed unexpected depths of reflection.

\section{Should others follow our example on psychodynamic groups?}

We would like to stress the importance of providing a psychological intervention for psychosis, particularly in light of the new data on childhood trauma and neglect in psychosis (Ross 2008; Read 2013). Much of this research work into psychotherapy for psychosis refers to the early stages of the disorder; we are keen to use principles from this early intervention practice and apply them to patients with more chronic disorder. Indeed, authors have highlighted the importance of doing more group work for patients affected by psychotic disorders in both the community and in-patient settings (Radcliffe 2007; Canete 2012).

Having said that, beyond the voluntary attendance, it is difficult to prove the effectiveness of this intervention in an evidence-based fashion. We do not know whether regular attendance at our group reduces rates of hospital admission, improves psychometric scores or adherence to medication, or has any other statistically significant positive outcome. However, we were struck by the powerful stories that unfolded in the group from a sample of patients who have long been excluded from psychotherapy. Clinical management of patients affected by treatment-resistant schizophrenia, by definition, operates beyond the realm of evidence, for psychotherapy as well as for medication and social intervention.

\section{Conclusions}

We found that a psychodynamic psychotherapy group for patients with chronic schizophrenia is possible and meaningful: patients came, talked and reflected. It addresses some important deficits in the type of psychological interventions we provide for management of schizophrenia. We have to bear in mind that measuring the benefits of such interventions is particularly difficult. The deficits in emotional understanding (empathy) can be so severe in schizophrenia that patients may not be able to relinquish a very concrete internal world. Despite this, emotions can surface in the group and be processed using the group container. We had anticipated managing a room full of psychotic material, but in fact the group thought about material in terms of human experience, looking for connections within and between experiences. Ultimately, the unstructured setting offered an opportunity for patients to find a meaningful way to use the space, where other structured therapeutic spaces have lost their meaning.

We believe that offering containment, openness and time are crucial therapeutic factors in working with this group of patients and we are confident that all psychotherapy group interventions for chronic psychosis, including CBT tailored for chronic symptoms and self-help groups for psychosis, can benefit from adapting these elements in their intervention.

\section{References}

Bion WR (1957) Differentiation of the psychotic from non-psychotic personalities. International Journal of Psychoanalysis, 38: 266-75.

Bridle D, McCabe R, Priebe S (2013) Incorporating psychotherapeutic methods in routine community treatment for patients with psychotic disorders. Psychosis, 5: 154-65.

Canete M, Ezquerro A (2012) Bipolar affective disorders and group analysis. Group Analysis, 45: 203-17.

Chazan R (1993) Group analytic therapy with schizophrenic outpatients. Group, 17: 164-78.

de Bosset $F$ (1991) Group psychotherapy in chronic psychiatric outpatients: a Toronto model. International Journal of Group Psychotherapy, 41: 65-78.

de Chavez MG (2000) Comparative study of the therapeutic factors of group therapy in schizophrenic inpatients and outpatients. Group Analysis, 33: 251-64.

Foulkes SH, Anthony EJ (1957) Group Psychotherapy: The Psychoanalytic Approach. Karnac Books.

Freud S (1914) On narcissism: an introduction. Reprinted in The Standard Edition of the Complete Psychological Works of Sigmund Freud (trans \& ed J Strachey), vol 14: 67-102. Hogarth Press. 
Fromm-Reichmann F (1948) Notes on the development of treatment of schizophrenics by psychoanalytic psychotherapy. Psychiatry, 11: 263-73.

Gabrovsek VP (2009) Inpatient group therapy of patients with schizophrenia. Psychiatria Danubina, 21 (suppl 1): 67-72.

Haker H, Rössler W (2009) Empathy in schizophrenia: impaired resonance. European Archives of Psychiatry and Clinical Neuroscience, 259: 352-61.

Hughes P, Kerr I (2000) Transference and countertransference in communication between doctor and patient. Advances in Psychiatric Treatment, 6: 57-64.

Jauhar S, McKenna PJ, Radua J, et al (2014) Cognitive-behavioural therapy for the symptoms of schizophrenia: systematic review and metaanalysis with examination of potential bias. British Journal of Psychiatry, 204: $20-9$

Jones C, Hacker D, Cormac I, et al (2012) Cognitive behaviour therapy versus other psychosocial treatments for schizophrenia. Cochrane Database of Systematic Reviews, 4: CD008712.

Kanas N (1986) Group therapy with schizophrenics: a review of controlled studies. International Journal of Group Psychotherapy, 36: 339-51.

Kanas N (1996) Group Therapy for Schizophrenic Patients. American Psychiatric Press.

Lucas R (2008) The psychotic wavelength. Psychoanalytic Psychotherapy, 22: $54-63$

Martindale BV (2007) Psychodynamic contributions to early intervention in psychosis. Advances in Psychiatric Treatment, 13: 34-42

Maxmen JS (1973) Group therapy as viewed by hospitalized patients. Archives of General Psychiatry, 28: 404-8.

McCormick LM, Brumm MC, Beadle JN, et al (2012) Mirror neuron function, psychosis, and empathy in schizophrenia. Psychiatry Research: Neuroimaging, 201: 233-9.

National Institute for Health and Care Excellence (2014) Psychosis and Schizophrenia in Adults: Treatment and Management (NICE Clinical Guidelines CG178). NICE.

Penn DL, Sanna LJ, Roberts DL (2008) Social cognition in schizophrenia: an overview. Schizophrenia Bulletin, 34: 408-11.
Radcliffe J, Smith R (2007) Acute in-patient psychiatry: how patients spend their time on acute psychiatric wards. Psychiatric Bulletin, 31: 167-70.

Read J, Dillon J (2013) Models of Madness: Psychological, Social and Biological Approaches to Psychosis (2nd edn). Brunner-Routledge.

Reininghaus UA, Morgan C, Simpson J, et al (2008) Unemployment, social solation, achievement-expectation mismatch and psychosis: findings from the AESOP Study. Social Psychiatry and Psychiatric Epidemiology, 43: $743-51$.

Rosenbaum B, Valbak K, Harder S, et al (2005) The Danish National Schizophrenia Project: prospective, comparative longitudinal treatment study of first-episode psychosis. British Journal of Psychiatry, 186 394-9.

Rosenbaum B, Martindale B, Summers A (2013) Supportive psychodynamic psychotherapy for psychosis. Advances in Psychiatric Treatment, 19: $310-8$.

Rosenfeld HA (1965) Psychotic States: A Psychoanalytical Approach. Hogarth Press.

Ross CA (2008) Dissociation and psychosis: conceptual issues. Journal of Psychological Trauma, 6 (2-3): 21-34.

Seikkula J, Aaltonen J, Alakare B, et al (2006) Five-year experience of first-episode nonaffective psychosis in open-dialogue approach: treatment principles, follow-up outcomes, and two case studies. Psychotherapy Research, 16: 214-28.

Smith J (1999) Five questions about group therapy in long-term schizophrenia. Group Analysis, 32: 515-24.

Tarrier N, Haddock G, Barrowclough C, et al (2002) Are all psychological treatments for psychosis equal? The need for CBT in the treatment of psychosis and not for psychodynamic psychotherapy. Psychology and Psychotherapy, 75: 365-79.

Turkington D, McKenna PJ (2003) Is cognitive-behavioural therapy a worthwhile treatment for psychosis? British Journal of Psychiatry, 182 . 477-9.

Yalom ID (1983) Inpatient Group Psychotherapy. Basic Books.

\section{MCOs}

Select the single best option for each question stem

\section{A key feature of a psychodynamic group for psychosis is:}

a an unstructured setting

b material for discussion brought by facilitators

c fixed duration of therapy

d requirement to sign a pre-treatment contract

e use of coping strategies.

\section{One example of an adaptation of the} psychodynamic setting to psychosis is:

a use of systematic interpretation of psychotic material

b a more teaching role for facilitators

c high level of demand on patients

d open attendance

e assigning specific tasks to each participant at the beginning of each session.

\section{Which of the following was not an} outcome of our group experience?

a regular attendance for a core number of patients

b emotions expressed despite concrete thinking

c patients became aware of the regular time and place of the group

d reduction of positive psychotic symptoms

e expression of empathy and involvement among participants.

\section{Concrete thinking:}

a was an obstacle to engagement with psychodynamic psychotherapy

b predicted disengagement from therapy in our group

c was a useful approach for processing emotional difficulties

$\mathrm{d}$ is never manifestation of chronic schizophrenia

e was completely relinquished at the end of the treatment.
5 A psychodynamic approach in psychosis

a is supported by strong evidence

b can have a role in treatment with necessary adaptation

c is always contraindicated

$d$ has only been used in Scandinavian countries

$\mathrm{e}$ is only effective when applied according to the rules of classic Freudian psychoanalysis. 